Annual Research \& Review in Biology

9(2): 1-8, 2016, Article no.ARRB.22895

ISSN: 2347-565X, NLM ID: 101632869

SCIENCEDOMAIN international

www.sciencedomain.org

\title{
Phylogenetic Considerations in the Evolutionary Development of Aminoglycoside Resistance Genes in Pathogenic Bacteria
}

\author{
Tommy Rodriguez ${ }^{1 *}$ \\ ${ }^{1}$ Department of Research and Development, Pangaea Biosciences, Miami, FL, USA.
}

Author's contribution

The sole author designed, analyzed and interpreted and prepared the manuscript.

Article Information

DOI: 10.9734/ARRB/2016/22895

Editor(s):

(1) George Perry, Dean and Professor of Biology, University of Texas at San Antonio, USA.

Reviewers:

(1) Hazem Mohammed Ebraheem Shaheen, Damanhour University, Damanhour, Egypt.

(2) Anonymous, University of Sao Paulo, Brazil.

Complete Peer review History: http://sciencedomain.org/review-history/12792

Case Study

Received $3^{\text {rd }}$ November 2015

Accepted $15^{\text {th }}$ December 2015

Published 25 $5^{\text {th }}$ December 2015

\begin{abstract}
This study revisits antibiotic resistance as a source of evolutionary development in pathogenic bacteria. By taking a molecular phylogenetic approach to this inquiry, I seek to find homologous correlations in antimicrobial resistance gene families across a broad spectrum of bacteria, as to identify the possible acquisition of those genes through divergent events in evolutionary context. In order to test this, I examine the various degrees of genetic similarity in two antimicrobial resistance genomic datasets, namely aadA1 \& aadA2 aminoglycoside resistance genes, among bacteria that occur in a multitude of environments. Moreover, the results from phylogenetic analysis suggests that pathogenic antibiotic resistance for aadA1 \& aadA2 aminoglycoside resistance genes may have been acquired through evolutionary events with a common ancestor of a soil-dwelling bacterium.
\end{abstract}

Keywords: Bacteria; pathogens; phylogeny; molecular phylogenetics; aminoglycoside resistance genes; antimicrobial resistance; multiple sequence alignment; comparative genomics. 


\section{INTRODUCTION}

Antibiotic resistance in pathogenic bacteria has been the source of concern in recent times. Each year in the United States, at least 2 million people become infected with bacteria that are resistant to antibiotics and at least 23,000 people die each year as a direct result of these infections [1,2]. Repeated usage of antibiotic drugs can cause resistance to become more prevalent. Susceptible bacteria are killed or inhibited by an antibiotic, resulting in a selective pressure for the survival of resistant strains [3,4]. Moreover, resistance is rapidly expanding to include several critical antimicrobials used to treat the most invasive infections.

Today, new findings suggest that antibiotic resistance appeared long before the introduction of antibiotic drugs [5]. Over three hundred sets of homologous protein coding genes for antimicrobial resistance have been identified among the five bacteria types [6].

Interestingly, some of the highest degrees of genetic similarity in antimicrobial resistance genes are shared between bacterial pathogens and modern soil-dwelling varieties. Nitrogenfixing bacteria are thought to have developed resistance from selective pressures in soil, which acts as a reservoir for antimicrobial resistance. Such a scenario presumes that, pathogenic bacteria may have acquired resistance through evolutionary events with a common ancestor of a soil-dwelling bacterium.

This paper revisits antibiotic resistance as a source of evolutionary development in pathogenic bacteria. By taking a molecular phylogenetic approach to this inquiry, I seek to find homologous correlations in antimicrobial resistant gene families across a broad spectrum of bacteria, as to indentify the possible acquisition of those genes through divergent events in evolutionary context. The scope of my investigation will again feature techniques in comparative genomics for reconstructing a phylogeny based on two distinct sets of multiple sequence alignments involving antimicrobial resistance genes.

\subsection{Antimicrobial Resistance Gene Families}

The ARDB (Antibiotic Resistance Genes Database) lists approximately three hundred seventy three protein coding genes for antimicrobial resistance [6]. A significant percentage of those genes are associated with pathogenic bacteria. This report concerns itself with one group, of one particular variety: aminoglycoside resistance genes. Aminoglycoside resistance genes are widely spread in bacteria genera, and they play an important role in antibiotic drug resistance. These particular genes are characterized by three primary mechanisms of resistance, namely ribosome alteration, decreased permeability, and inactivation of the antibiotics by modifying enzymes $[7,8]$.

Antimicrobial resistance spreads as bacteria themselves move from place to place. For decades, soil ecologists have speculated that soil acts as a reservoir for antimicrobial resistance [9]. Presumably, nitrogen-fixing bacteria have evolved the ability to become antimicrobial resistance as a countermeasure to naturally occurring environmental threats, such as the compounds frequently produced by competing microbes. As preliminary data indicates, pathogenic and nitrogen-fixing bacteria possess a similar genetic basis for resistance but do not share an obvious means for transfer among themselves. A 2012 paper entitled, "The shared antibiotic resistome of soil bacteria and human pathogens," elaborates on the significance of sequence similarities across different bacteria species that occur in a host of different environments [10]. In this study, Forsberg et al. demonstrated a high degree of matching DNA sequences between soil-dwelling and pathogenic bacteria, and it provides evidence for the exchange of antimicrobial resistance genes between environmental bacteria and clinical pathogens [10].

To further support these findings, a more recent study shows that antimicrobial resistance genes found in the human microbiota must have also developed prior to synthetic and semi-synthetic antibiotics. The study identified a number of antimicrobial resistance genes in the human microbiota that are targeted at natural antibiotics of the sort produced by soil microbes [11]. Moreover, I hold the viewpoint shared among others that soil-dwelling bacteria may be the original source of antibiotic resistance in bacterial pathogens. In order to test this, I will examine the various degrees of genetic similarity in selected aminoglycoside resistance genes among a broad spectrum of bacteria that occur in different environments. 


\section{MATERIALS AND METHODS}

\subsection{Gene Selection}

This investigation utilizes two partial sets of aminoglycoside resistance genes [Aminoglycoside O-nucleotidylyltra] (aadA1 \& aadA2) for comparative analysis. Aminoglycoside resistance genes are ideal genomic candidates, as they encompass a broad antimicrobial spectrum shared between diverse populations of bacteria [12]. These gene families are also generally associated with an exceptionally highlevel of resistance to antibiotics. The mechanisms that modify aminoglycosides by adenylylation [in Aminoglycoside Onucleotidylyltra] are most notably known to occur in response to antibiotic complex produced by Streptomyces kanamyceticus from soil [6].

The bacterial species appropriated for this study are found to contain resistance strains of these naturally occurring responses. As such, I compiled two distinct FASTA data files containing a combination of eleven nucleotide sequences derived from pathogenic bacteria and soildwelling varieties. I ran several BLAST similarity searches against Salmonella enterica subspecies strain SRC54 [13-16], and this procedure returned a significantly high number of homologous sequences to be later used in this study. ${ }^{2}$ Each nucleotide sequence was obtained via NCBI [nucleotide] database archives. See table 1 for accession numbers.

\subsection{Multiple Sequence Alignment}

Before outlining this phase of my investigation, I should briefly note that four of my sequence selections required a reverse protein-DNA translation (as highlighted in Table 1 by an asterisk). This procedure was conducted using SMS Format Conversion. ${ }^{2}$ See references for a complete documentation. Furthermore, as with other case studies that I have conducted involving similar frameworks, this investigation also required a series of multiple sequence alignment (MSA) operations in preparation for phylogenetic reconstruction. For purposes of obtaining the most highly-accurate base-pair alignments possible, I selected Kalign for multiple sequence alignment; an accurate and fast MSA algorithm [17]. Kalign is an extension of Wu-Manber approximate pattern-matching algorithm, which is based on Levenshtein distances. This strategy enables Kalign to estimate sequence distances faster and more accurately than other popular iterative methods. Comparisons done by Lassmann and Sonnhammer [17] show that Kalign is about 10 times faster than ClustalW and, depending on the alignment size, up to 50 times faster than other iterative methods; Kalign also delivers better overall resolution.

Kalign is renowned for producing optimal execution times, and this procedure would require minimal computational resources. First, I initiated UGENE's multiple sequence alignment tool by importing and processing two distinct gene family datasets [in FASTA format], each containing eleven nucleotide sequences. ${ }^{2}$ Kalign for MSA gap penalty scores were modified slightly during successive intervals until an optimal global alignment was achieved. The first set of intervals resulted in a 2,714 base-pair alignment, whereas the second produced a 2,226 base-pair alignment.

Table 1. Bacteria identification and sequence data accession numbers

\begin{tabular}{lll}
\hline Bacteria Identification & Aada1 FASTA description & Aada2 FASTA description \\
\hline Salmonella enterica & $>$ gi|261347676|gb|GQ924769.1 & $>$ gi|112950028|gb|DQ836009.1 \\
Escherichia coli & $>$ gi|925216761|gb|KR028103.1 & $>$ gi|385282937|gb|JQ414042.1 \\
Leclercia adecarboxylata & $>$ gi|695227314|ref|NG_041647.1* & $>$ gi|723217856|gb|KM278190.1 \\
Aeromonas hydrophila & $>$ gi|723217868|gb|KM278193.1 & $>$ gi|723217859|gb|KM278189.1 \\
Riemerella anatipestifer & $>$ gi|350281978|gb|JF920804.1 & $>$ gi|63192146|gb|AY968682.1 \\
Comamonas testosteroni & $>$ gi|723217875|gb|KM278197.1 & $>$ gi|723217853|gb|KM278191.1 \\
Citrobacter freundii & $>$ gi|409183968|gb|JX494725.1 & $>$ gi|5881157|gb|AF175203.1 \\
M.esteraromaticum & $>$ gi|636631991|gb|KJ575540.1* & $>$ gi|636631991|gb|KJ575540.1 \\
Laribacter hongkongensis & $>$ gi|297578538|gb|GU726913.1 & $>$ gi|297578519|gb|GU726907.1 \\
Aeromonas caviae & $>$ gi|197244601|emb|FM207629.1 & $>$ gi|636631949|gb|KJ568502.1 \\
Providencia stuartii & $>$ gi|410691328|ref|NC_019375.1* & $>$ gi|410691328|ref|NC_019375.1* \\
\hline
\end{tabular}




\subsection{Building a Phylogeny Based on Aminoglycoside Resistance Genes}

Going forward, a phylogenetic reconstruction would solidify my aadA1 \& aadA2 base-pair alignments into a meaningful, workable diagram. This portion of the study becomes imperative to my original hypothesis that soil-dwelling bacterium may be the original source of certain types of antibiotic resistance in bacterial pathogens; thus, special consideration was given toward algorithmic selection for phylogenetic reconstruction. Here, I implemented PHYLIP neighbor-joining method coupled with distance matrix model F84 on both sets of base-pair alignments. This procedure would also require additional bootstrapping compilers to help evaluate the strengths of the inner and outer nodes ${ }^{2}$.

The PHYLIP neighbor-joining algorithm is suitable for generating highly probable diagrams in scenarios involving low degrees of variance, regardless of dataset size. An accurate and statically consistent polynomial-time algorithm, PHYLIP neighbor-joining does not assume that all lineages evolve at the same rate, and it constructs a tree by successive clustering of lineages, setting branch lengths as the lineages join [where a set of $n$ taxa requires $n-3$ iterations; each step is repeated by $(n-1) \times(n-$ 1)] [18]. For illustration purposes, the following formulas demonstrate a standard neighborjoining Q-matrix algorithm, as the type used in this study:

$$
\begin{aligned}
& Q(i, j)=(n-2) d(i, j)-\Sigma\{n, k=1\} d(i, k)-\Sigma \\
& \{n, k=1\} d(j, k)
\end{aligned}
$$

Pair to node (distances):

$$
\begin{aligned}
& (f, u)=1 / 2 d(f, g)+1 / 2(n-2)\left[\sum\{n, k=1\} d(f, k)\right. \\
& \left.-\sum\{n, k=1\} d(g, k)\right]
\end{aligned}
$$

Taxa to node (distances):

$$
d(u, k)=1 / 2[d(f, k)+d(g, k)-d(f, g)]
$$

\section{RESULTS}

\subsection{Sequence Analysis \& Phylogenetic Reconstruction}

Among the eleven bacterial strains included in each gene family subset, the sequence similarity percentage between them averaged $78.6 \%$ and $82.6 \%$, respectively, with values ranging from $54 \%$ to $99 \%$. Five pathogenic strains yielded exceptionally high sequence similarity ratios, ranging from $96 \%$ to $99 \%$ and $98 \%$ to $99 \%$, respectively. Based on these estimates, five sequences could be assigned to a subgroup of very closely related strains. It is generally admitted that sequences with greater than $97 \%$ identity are typically assigned to the same species, those with $>95 \%$ identity are typically assigned to the same genus, and those with $>80 \%$ identity are typically assigned to the same phylum [19]. However, due to partial sequence sizes, the latter may not apply here. See Table 2.

Subsequently, one might then project a subgroup consisting of five highly homologous sequences to dictate the trajectory of clade positioning within each tree, beginning with its inner node(s) and extending outward. And this pattern, indeed, highlighted the lineage disbursements in each diagram, where five of eleven highly homologous sequences fell within the closest proximity of all sequence candidates. As Fig. 2 illustrates, the

Table 2. aadA1 and aadA2 sequence similarity ratios

\begin{tabular}{llll}
\hline Species Name & Aada1 & Aada2 & Sequence similarity ratio \\
\hline Salmonella enterica & 0.99 & 0.99 & 0.99 \\
Escherichia coli & 0.84 & 0.98 & 0.91 \\
Leclercia adecarboxylata & 0.78 & 0.99 & 0.885 \\
Aeromonas hydrophila & 0.73 & 0.99 & 0.86 \\
Riemerella anatipestifer & 0.96 & 0.88 & 0.92 \\
Comamonas testosteroni & 0.74 & 0.99 & 0.865 \\
Citrobacter freundii & 0.79 & 0.54 & 0.665 \\
M.esteraromaticum & 0.69 & 0.69 & 0.69 \\
Laribacter hongkongensis & 0.54 & 0.68 & 0.61 \\
Aeromonas caviae & 0.81 & 0.57 & 0.69 \\
Providencia stuartii & 0.78 & 0.79 & 0.785 \\
& 0.786 & 0.826 & 0.806 \\
\hline
\end{tabular}


taxon represented by the inner-most node(s) are assigned to species of clinical pathogens, collectively; whereas, strains positioned among the tree outgroups, occur in diverse environments (sewage, soil \& water); including one exclusively soil-dwelling strain (Comamonas testosteroni) [20,21].

The analysis of aadA1 sequences reveal rooting inconsistencies with that found in Fig. 2. Furthermore, among the sister groups located within the inner-most nodes remain three clinical pathogens that correspond to Fig. 2. As we move outward from one external node to the next, the arrangement of taxa becomes less distinguishable. This discrepancy is also featured in the similarity ratios shown in Table 2 , and the nucleotide substitution rates on the area graphs shown in Figs. 1 (A) and Fig. 2 (B).

Yet, despite the inherit differences between them, an underlying trend was identified: (a) positioning among the inner-most and outer-most taxon on each diagram - namely clinical pathogens and soil-dwelling strains, respectively - correlate on both instances; (b) soil-dwelling taxa, represented by their position along the outgroups of each tree, appear having older lineages for aadA1 \& aadA2 aminoglycoside resistance genes. And thus, by assessing the units of branch length on both diagrams, where the sequence candidates with higher nucleotide substitution rates reside on the far ends, I find very good support for the precursors of aadA1 \& aadA2 aminoglycoside resistance genes in pathogens. The significance of these results also provides evidence for the exchange of antimicrobial resistance genes across different hosts, environments and geographical origins. See Fig. 1 (A) and Fig. 2 (B) for more details.

\section{DISCUSSION}

Antimicrobial resistance in bacteria is not a modern evolutionary innovation. In fact, antibiotics made from compounds produced by bacteria and fungi have existed long before humans formulated the first antibiotic drugs.

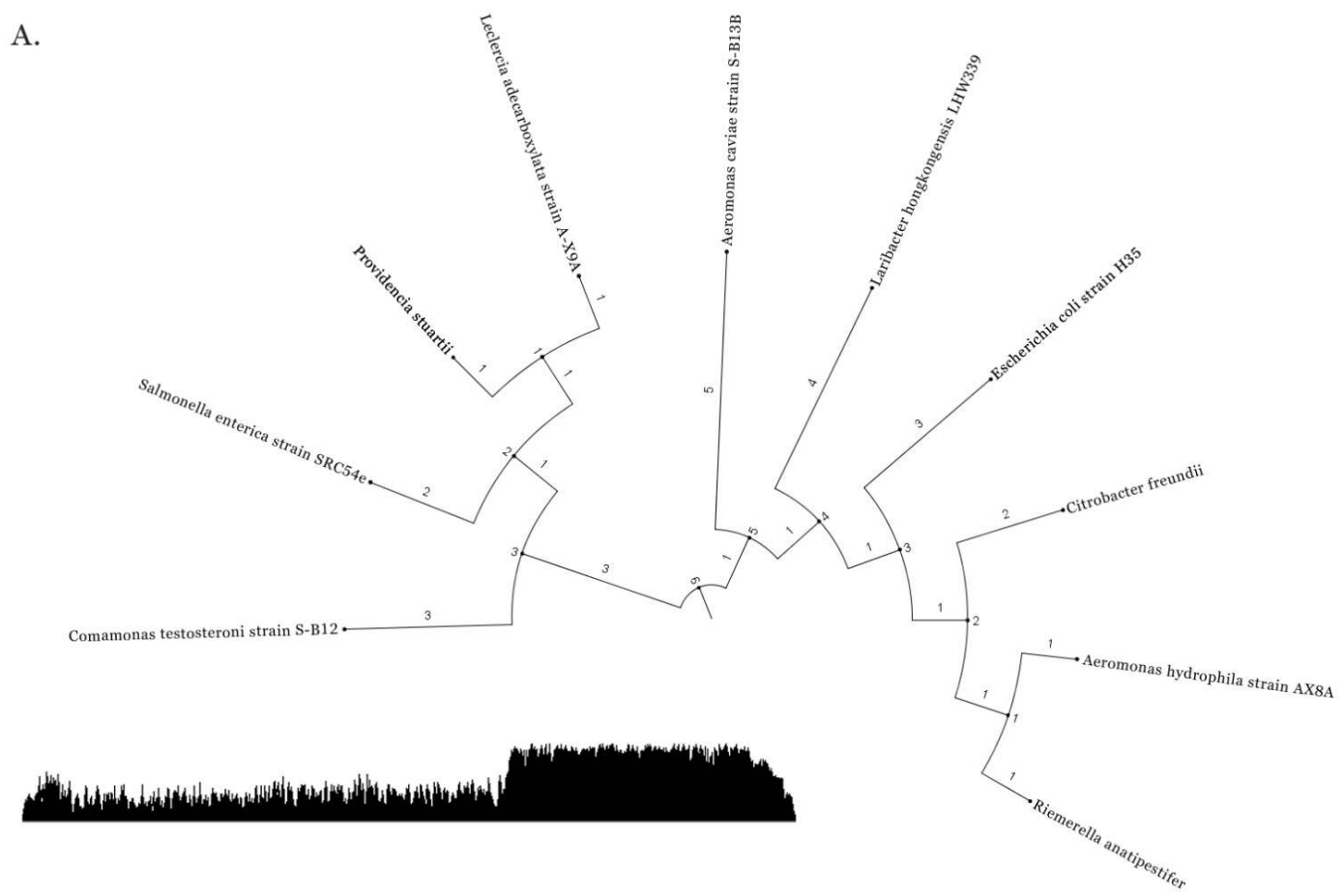

Fig. 1. (A) Phylogenetic reconstruction of aadA1 aminoglycoside resistance genes. Taxa order (inner-node to outer-node arrangement): clade a) Leclercia adecarboxylataA-X9A, Providencia stuartii, Salmonella enterica SRC54e, Comamonas testosteroni S-B12; clade b) Riemerella anatipestifer, Aeromonas hydrophila AXBA, Citrobacter freundii, Escherichia coli H35, Laribacter hongkongensis LHW339, Aeromonas caviae S-B13B. Nucleotide substitution area graph included 


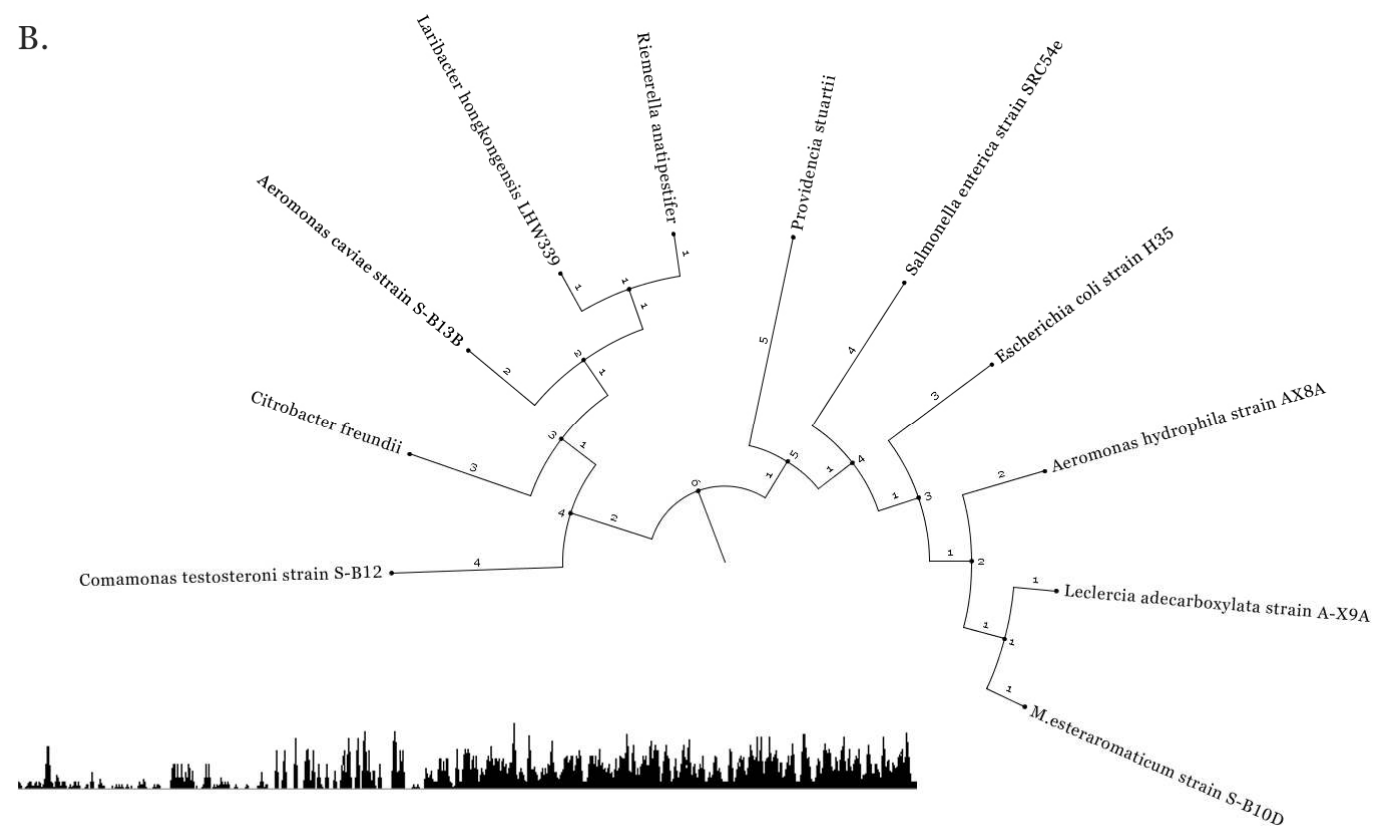

Fig. 2. (B) Phylogenetic reconstruction of aadA2 aminoglycoside resistance genes. Taxa order (inner-node to outer-node arrangement): clade a) Riemerella anatipestifer, Laribacter hongkongensis LHW339, Aeromonas caviae S-B13B, Citrobacter freundii, Comamonas testosteroni S-B12; clade b) M.esteraromaticum S-B10D, Leclercia adecarboxylataA-X9A, Aeromonas hydrophila AXBA, Escherichia coli H35, Salmonella enterica SRC54e, Providencia stuartii. Nucleotide substitution area graph included

In nature, antibiotics can increase selective pressure in a population of bacteria, promoting resistant bacteria by supporting its survival prospects. And, as it occurs in the medical sector, antibiotic drugs are used too often or incorrectly, which can cause resistance to spread faster than it would in natural settings [7,22-24]. For this reason, a focus on identifying the evolutionary events that led to the acquisition of resistance in pathogens could help us better understand the interactions that occur between diverse bacteria across a wide range of hosts and environments.

Bacteria use horizontal gene transfer as one primary method for exchanging genetic information. It is also known that recombination plays an important evolutionary role [25]. Although naturally occurring genetic mutations in bacterium can create the variation needed to produce new genes for antimicrobial resistance within a population, it is more likely that acquired resistance via DNA transfer between different strains would best explain the homologous correlations observed in antimicrobial resistance gene families. High sequence similarity ratios in
aadA1 \& aadA2 aminoglycoside resistance genes among distinct species also imply that DNA transfer has occurred between ancestral organisms in the past.

Consequently, Forsberg et al. points out that whether shared resistance is confined to genes of particular mechanisms or applies to many genes with diverse mechanisms of resistance is still unknown [10]. Whether a single horizontal gene transfer event between environment and clinic can result in the de novo acquisition of a multidrug-resistant phenotype is [also] unclear [10]. Thus, looking at my results hereafter, it is difficult to speculate on how likely or unlikely each scenario may be; especially when this investigation did not involve full-length genomic datasets, but two partial sequences belonging to one gene family from eleven species. In any case, I would simply stress the scope of this study is not to speculate on the mechanisms for acquisition, but rather to illustrate a phylogeny based on relevant genes for antimicrobial resistance. In that such case, I have demonstrated that pathogenic antibiotic resistance for aadA1 \& aadA2 aminoglycoside 
resistance genes may have been acquired through evolutionary events with a common ancestor of a soil-dwelling bacterium.

\section{CONCLUSION}

The exchange of resistance between pathogens and soil-dwelling bacteria emphasizes the clinical importance of the soil resistome [10]. From a phylogenetic perspective, this study reinforces the inferences already reached by others. Based on my results, I find very good support for the precursors of aadA1 \& aadA2 aminoglycoside resistance genes in pathogens. My results also provide evidence for the exchange of aadA1 \& aadA2 aminoglycoside resistance genes across different hosts, environments and geographical origins.

Lastly, it should be noted that a phylogenetic reconstruction involving two partial genomic datasets from eleven distinct species does not substantially improve on the antibiotic resistome as a whole. As others have pointed out, determining the clinical impact of environmental resistance requires a deeper profiling of environmental reservoirs for the organisms and genotypes most likely to exchange resistance with pathogenic varieties [10]. I too propose a more thorough investigation, as to include a wider range of species and antimicrobial resistance gene families.

\section{ADDITIONAL NOTES}

${ }^{2}$ UGENE was used in comparative sequence analysis. The DNA sequences noted above were in FASTA format. They were all obtained from the NCBI database archives. SMS Format Conversion is available at www.bioinformatics.org.

\section{COMPETING INTERESTS}

Author has declared that no competing interests exist.

\section{REFERENCES}

1. Anonymous. Salmonella (non-typhoidal). World Health Organization; 2013.

Available:http://www.who.int/mediacentre/f actsheets/fs139/en/ (Accessed June 2015)

2. Jackson BR, Griffin PM, Cole D, Walsh KA, Chai SJ. Outbreak-associated Salmonella enterica serotypes and food commodities, United States, 1998-2008. Emerging Infectious Diseases. 2013;19(8):1239

3. Anonymous. General Background: About antibiotic resistance. Tuffs University; 2015.

Available:http://www.tufts.edu/med/apua/a bout issue/about antibioticres.shtml (Accessed July 2015)

4. Seyfarth AM, Wegener HC, Frimodt-Møller N. Antimicrobial resistance in Salmonella enterica subsp. enterica serovar typhimurium from humans and production animals. Journal of Antimicrobial Chemotherapy. 1997;40(1):67-75.

5. Anonymous. Antibiotic-resistant bacteria were around a long time before our antibiotics. Motherboard; 2015.

Available:http://motherboard.vice.com/read lantibiotic-resistant-bacteria-were-arounda-long-time-before-our-antibiotics-2 (Accessed June 2015)

6. Anonymous. ARDB - Antibiotic Resistance Genes Database. University of Maryland; 2015.

Available:http://ardb.cbcb.umd.edu/ (Accessed March 2015)

7. Anonymous. Aminoglycoside resistance. Belgium Biosafety Server; 2015.

Available:http://www.antibioresistance.be/a minoglycosides.html (Accessed June 2015)

8. Barnhart C. Mechanisms of aminoglycoside resistance. University Of Pennsylvania; 2002.

9. Williams S. soil may be source of drugresistant bacteria. Science Magazine; 2012.

10. Forsberg KJ, Reyes A, Wang B, Selleck EM, Sommer MO, Dantas G. The shared antibiotic resistome of soil bacteria and human pathogens. Science. 2012; 337(6098):1107-11.

11. Clemente JC, Pehrsson EC, Blaser MJ, Sandhu K, Gao Z, Wang B, Magris M, Hidalgo G, Contreras M, Noya-Alarcón Ó, Lander $\mathrm{O}$. The microbiome of uncontacted Amerindians. Science Advances. 2015; 1(3):e1500183.

12. Nie L, Lv Y, Yuan $M$, Hu $X$, Nie T, Yang $X$, Li G, Pang J, Zhang J, Li C, Wang X. Genetic basis of high level aminoglycoside resistance in Acinetobacter baumannii from Beijing, China. Acta Pharmaceutica Sinica B. 2014;4(4):295-300.

13. Andino A, Hanning I. Salmonella enterica: Survival, colonization, and virulence 
differences among serovars. The Scientific World Journal; 2015.

14. DiMarzio M, Shariat N, Kariyawasam S, Barrangou R, Dudley EG. Antibiotic resistance in Salmonella enterica serovar Typhimurium associates with CRISPR sequence type. Antimicrobial Agents and Chemotherapy. 2013;57(9):4282-9.

15. McClelland M, Sanderson KE, Spieth J, Clifton SW, Latreille P, Courtney L, Porwollik S, Ali J, Dante M, Du F, Hou S. Complete genome sequence of Salmonella enterica serovar Typhimurium LT2. Nature. 2001;413(6858):852-6.

16. Porwollik S, Boyd EF, Choy C, Cheng P, Florea L, Proctor E, McClelland M. Characterization of Salmonella enterica subspecies I genovars by use of microarrays. Journal of Bacteriology. 2004;186(17):5883-98.

17. Lassmann T, Sonnhammer EL. Kalign-an accurate and fast multiple sequence alignment algorithm. BMC bioinformatics. 2005;6(1):298.

18. Felsenstein J. Evolutionary trees from DNA sequences: A maximum likelihood approach. Journal of Molecular Evolution. 1981;17(6):368-76.

19. Chriki-Adeeb R, Chriki A. Bayesian phylogenetic analysis of rhizobia isolated from root-nodules of three Tunisian wild legume species of the genus Sulla. $J$ Phylogen Evolution Biol. 2015;3(149):2.
20. Abraham JM, Simon GL. Comamonas testosteroni bacteremia: A case report and review of the literature. Infectious Diseases in Clinical Practice. 2007;15(4):272-3.

21. Bayhan Gi, Tanır G, Karaman I, Özkan Ş. Comamonas testosteroni: An unusual bacteria associated with acute appendicitis. Balkan Medical Journal. 2013;30(4):447.

22. Conlan S, Thomas PJ, Deming C, Park M, Lau AF, Dekker JP, Snitkin ES, Clark TA, Luong K, Song Y, Tsai YC. Singlemolecule sequencing to track plasmid diversity of hospital-associated carbapenemase-producing Enterobacteriaceae. Science Translational Medicine. 2014;6(254):254ra126.

23. Parry CM. Antimicrobial drug resistance in Salmonella enterica. Current opinion in infectious diseases. 2003;16(5):467-72.

24. Rabsch W, Andrews HL, Kingsley RA, Prager R, Tschäpe H, Adams LG, Bäumler AJ. Salmonella enterica serotype Typhimurium and its host-adapted variants. Infection and Immunity. 2002; 70(5):2249-55.

25. Didelot $X$, Bowden R, Street T, Golubchik T, Spencer C, McVean G, Sangal V, Anjum MF, Achtman M, Falush D, Donnelly P. Recombination and population structure in Salmonella enterica. PLoS Genet. 2011;7(7):e1002191.

(0) 2016 Rodriguez; This is an Open Access article distributed under the terms of the Creative Commons Attribution License (http://creativecommons.org/licenses/by/4.0), which permits unrestricted use, distribution, and reproduction in any medium, provided the original work is properly cited.

Peer-review history:

The peer review history for this paper can be accessed here: http://sciencedomain.org/review-history/12792 